\title{
Fitoplancton del río Tehuantepec, Oaxaca, México y algunas relaciones biogeográficas
}

\author{
José L. Moreno-Ruiz ${ }^{1-4}$, Margarito Tapia-García ${ }^{1}$, Ma. Del Carmen González-Macias ${ }^{3}$ \& \\ Ma. Guadalupe Figueroa-Torres ${ }^{4}$ \\ 1 Universidad Autónoma Metropolitana-Iztapalapa, C.P. 09340, A.P. 55-535, México D.F., México; \\ jlmr64@yahoo.com; mtg@xanum.uam.mx \\ 2 Instituto de Geología, UNAM, México D.F., México. \\ 3 Instituto Mexicano del Petróleo, Subdirección de Protección Ambiental, Estudios Ecológicos, C.P. 07730, A.P. \\ 14-805, México D.F., México; magonzal@www.imp.mx \\ 4 Universidad Autónoma Metropolitana-Xochimilco, Ficología, C.P. 04960, México D.F., México; \\ figueroa@correo.xoc.uam.mx
}

Recibido 02-XII-2005. Corregido 05-III-2007. Aceptado 28-VIII-2007.

\begin{abstract}
Phytoplankton from Tehuantepec River, Oaxaca, Mexico and some biogeographical relationships. Phytoplankton species collected from the limnetic to euryhaline sections of Tehuantepec River were identified, classified and compared with regional information from Mexico and South America. We collected 15 samples every three months from July 1997 through August 1998 with a $20 \mu \mathrm{m}$ net and a Van Dorn bottle. Indicator values and a code checklist are included. A total of 58 families, 121 genera, 273 species, one subspecies, 75 varieties, 13 forms and one morphotype were identified in the taxa Bacillariophyta $(42.0 \%)$, Chlorophyta (29.0\%), Cyanoprocaryota (18.0\%), Euglenophyta (5.0\%), Dinophyta (3.0 \%), Cryptophyta (2.0 $\%)$ and Chrysophyta (1.0 \%). The predominant families were Scenedesmaceae (24 species), Oocystaceae (22), Bacillariophyceae (21), Chaetocerotaceae (15) and Euglenaceae (14). Five families, eight genera, 72 species, 45 varieties and eight forms are first records for Mexico. The species Chroococcus turgidus, Microcystis flosaquae and Pseudanabaena limnetica (which produce massive blooms or red tides) are important in this river of moderate water quality. Rev. Biol. Trop. 56 (1): 27-54. Epub 2008 March 31.
\end{abstract}

Key words: phytoplankton, neotropical, limnetic, euryhaline, indicator values, massive blooms, water quality.

El conocimiento del fitoplancton de agua dulce, salobre y marino en México aún es pobre a pesar de la existencia de antecedentes que datan del siglo XIX, y que se concentran principalmente en la región central del país. Asimismo, se han proporcionado varios listados florísticos en los que se incluyen algunos valores indicadores (Ortega et al. 1995, Moreno-Ruiz 2000, Moreno 2003), pero que omiten a muchas especies raras, por lo que todavía se requiere realizar el estudio de estas algas microscópicas para determinar su dinámica y comprender la complejidad de su estructura ecológica. Sin embargo, dada la amplitud y extensión del territorio mexicano, se necesita aplicar un enfoque regional para conocer éstas características ecológicas en una escala que suministre mayor precisión a las investigaciones que aquí se desarrollan (Moreno et al. 1996).

La subregión oaxaqueña presenta una gran problemática ambiental y hasta el momento, aún no se determinan todas las repercusiones sociales y ecológicas, que en el río Tehuantepec producen el desarrollo productivo, el crecimiento demográfico, las actividades industriales y los desastres naturales. El problema es delicado, si se considera también que es una zona pobremente estudiada respecto a sus recursos bióticos. Por lo anterior, en el presente trabajo se contribuye con el conocimiento 
de la composición del fitoplancton del río Tehuantepec, Oaxaca, se proporcionan datos de su distribución en México, en la región neotropical, valores indicadores ecológicos y un código para facilitar su manejo.

\section{MATERIALES Y MÉTODOS}

Descripción del área de estudio: el área de estudio se enmarca entre las coordenadas geográficas $16^{\circ} 10.405^{\prime}-16^{\circ} 22.741^{\prime} \mathrm{N}$ y 9507.99'-95¹7.425' W (Anónimo 1981), en el Istmo de Tehuantepec en la provincia denominada Planicie Costera Suroriental (Rzedowsky 1978). El clima es cálido subhúmedo con temperatura promedio de $27.3^{\circ} \mathrm{C}$, con la oscilación de las temperaturas medias anuales menores a $5{ }^{\circ} \mathrm{C}$ (García 1981). Existen dos épocas climáticas, la de sequía (noviembre a abril-mayo) y la de lluvias (mayo-junio a octubre). En la época de sequía y principalmente de enero a marzo, azotan fuertes vientos del norte denominados "Tehuantepecos o Tehuantepecanos" (Monreal-Gómez y Salas de León 1998). La limnología del río Tehuantepec muestra características heterogéneas, con valores elevados, similares a los que se han indicado en aguas continentales en áreas tropicales (Huszar et al. 1998). Con valores promedio de la profundidad en las épocas climáticas de sequía y lluvia de: 0.9-1.0 m, temperatura entre 26.7-30.5 ${ }^{\circ} \mathrm{C}$, salinidad con 2.381-2.137\%o, $\mathrm{pH}$ de 8.3-8.0, dureza $\left(\mathrm{CaCO}_{3}\right)$ 150.6-144.9 $\mathrm{mg}$, oxígeno 9.557-10.185 ppm, amoniaco 0.050-0.057 ppm, nitratos 0.193-0.539 ppm, nitrógeno total $0.615-4.663 \mathrm{ppm}$, ortofosfatos 0.025-0.060 ppm, fósforo total 0.042-0.097 ppm, relación N:P 12.192-74.156, y la clorofila $a$ con 2.996-7.012 $\mathrm{mgm}^{-3}$ (Anónimo 2000, Moreno-Ruiz et al., en prep.).

Se recolectaron 15 muestras de agua trimestralmente durante un año (julio 1997 a agosto 1998), en las dos márgenes y la parte central de cinco puntos distribuidos a lo largo de los últimos $30 \mathrm{~km}$ del río Tehuantepec. Los materiales se obtuvieron del nivel superficie con una red de $20 \mu \mathrm{m}$ de abertura de malla, y del nivel intermedio en la columna de agua con una botella Van Dorn de 21 de capacidad, mismos que se vaciaron en botellas de vidrio de $250 \mathrm{ml}$; se adicionó lugol como preservador. Asimismo, se limpió material con los métodos estándar referidos por Moreno (2003) para la elaboración de laminillas. Posteriormente se efectúo el análisis cualitativo y cuantitativo de materiales temporales por medio del vaciado de $2 \mathrm{ml}$ de cada muestra en una cubeta de sedimentación, así como la revisión de cada laminilla con el uso del fotomicroscopio invertido LEITZ-WEZLAR/DIAVERT y el barrido de transectos diametrales. Con la finalidad de realizar observaciones ultraestructurales se prepararon materiales adicionales (Moreno 2003) y se utilizaron los microscópios electrónicos de transmisión y barrido JEOL JEM-1200EX2 y JEOL JSM-5410LV, respectivamente.

La determinación, ordenación sistemática y los datos de la composición ecológica (valores indicadores) de los taxones ( $\mathrm{pH}$, salinidad, suministro metabólico de nitrógeno, relación nitrógeno total: fósforo total, saprobiedad, preferencia trófica, forma de vida, preferencia térmica, florecimientos, frecuencia o distribución y dominancia) se elaboraron con datos originales, de acuerdo a los planteamientos de Caljon (1983), Contreras-Espinosa et al. (1994), Van Dam et al. (1994), Moreno et al. (1996), Moreno-Ruiz (2000), Anónimo (2000), Siqueiros-Beltrones (2002) y Moreno (2003).

\section{Composición ecológica o valores indica-}

dores: la clasificación de los valores indicadores se presenta en el Cuadro 1. Con el promedio de los 11 valores de las variables originales (pH-dominancia) de cada taxon, se obtuvo el valor indicador ponderado individual.

Con el promedio del mayor valor que pueden tener las variables utilizadas, se obtuvo el mayor valor indicador de la calidad del agua (baja calidad del agua), la relación entre tres de este valor marcó el límite superior de la alta calidad del agua, y la multiplicación por tres de este último valor mostró el límite superior de la moderada calidad del agua. Los intervalos para este trabajo se proporcionan en el Cuadro 1. 


\section{CUADRO 1}

Clasificación de la composición ecológica o valores indicadores

TABLE 1

Classification of ecological composition or indicator values

Potencial de hidrógeno

1 acidobióntico, presencia óptima a $\mathrm{pH}<5.5$

2 acidófilo, presencia principalmente a $\mathrm{pH}<7$

3 circumneutral, presencia principal en valores de $\mathrm{pH}$ alrededor de 7

4 alcalífilo, presencia principal a $\mathrm{pH}>7$

5 alcalibióntico, presencia exclusiva a $\mathrm{pH}>7$

6 indiferente, sin óptimo aparente

Salinidad (modificada para caracterizar ecológicamente a los organismos limnéticos y salobres)

1 limnobionte estenohalino $<0.5$ ups

2 limnobionte eurihalino $0.5-8$ ups

3 limnobionte debilmente eurihalino o limnobionte eurihalino de primer grado 0.5-3 ups

4 limnobionte eurihalino de segundo grado 3-8 ups

5 hifalmirobionte, abunda en agua salobre y se presenta esporádicamente en el mar o agua dulce

6 salobre eurihalino 2-20 ups

7 salobre inferior o salobre inferior eurihalino 2-10 ups

8 salobre superior o salobre superior eurihalino 8-20 ups

9 marino eurihalino, vive comunmente en biotopos marinos

10 holeurihalino, existe en agua marina, salobre y en agua dulce

Suministro metabólico de nitrógeno (basado en la literatura y en la mayor concentración de nitrógeno total)

1 nitrógeno-autotrófico, tolera concentraciones muy pequeñas de nitrógeno

2 nitrógeno-autotrófico, tolera elevadas concentraciones de nitrógeno orgánico

3 nitrógeno-heterotrófico facultativo, necesita periodicamente elevadas concentraciones de nitrógeno orgánico

4 nitrógeno-heterotrófico obligado, necesita continuamente elevadas concentraciones de nitrógeno orgánico

\section{Relación nitrógeno total:fósforo total}

1 baja $<7$ (limitación de nitrógeno)

2 moderado, entre 15 y 7

3 alto $>15$ (limitación de fósforo)

Saprobiedad, de acuerdo a los valores sapróbicos individuales de los taxa

1 xenosapróbico $0-0.5$

2 oligosapróbico $0.51-1.5$

$3 \beta$-mesosapróbico 1.51-2.5 
CUADRO 1 (Continuación)

Clasificación de la composición ecológica o valores indicadores

TABLE 1 (Continued)

Classification of ecological composition or indicator values

$4 \alpha$-mesosapróbico 2.51-3.5

5 polisapróbico $3.51-4.0$

Preferencia trófica, según el índice trófico IT (basado en mg de clorofila a $/ \mathrm{m}^{3}$ )

Especies oligotróficas, habitan biotopos pobres en nutrimentos, equivalen a:

1 ultraoligotrófico $\left(0.000-0.122 \mathrm{mg} / \mathrm{m}^{3}\right) \mathrm{IT}=0-9$

$2 \alpha$-oligotrófico $\left(0.123-0.340 \mathrm{mg} / \mathrm{m}^{3}\right) \mathrm{IT}=10-19$

$3 \beta$-oligotrófico $\left(0.350-0.940 \mathrm{mg} / \mathrm{m}^{3}\right) \mathrm{IT}=20-29$

$4 \gamma$-oligotrófico $\left(0.950-2.600 \mathrm{mg} / \mathrm{m}^{3}\right) \mathrm{IT}=30-39$

mesotrófico, equivale a:

oligo-mesotrófico, con

$5 \alpha$-mesotrófico $\left(2.700-7.200 \mathrm{mg} / \mathrm{m}^{3}\right) \mathrm{IT}=40-49$

meso-eutrófico, con

$6 \beta$-mesotrófico $\left(7.300-20.000 \mathrm{mg} / \mathrm{m}^{3}\right) \mathrm{IT}=50-59$

especie eutrófica, habita biotopos ricos en nutrimentos, equivale a:

$7 \alpha$-eutrófico $\left(21.000-55.000 \mathrm{mg} / \mathrm{m}^{3}\right) \mathrm{IT}=60-69$

$8 \beta$-eutrófico $\left(56.000-155.000 \mathrm{mg} / \mathrm{m}^{3}\right) \mathrm{IT}=70-79$

9 -eutrófico $\left(156.000-425.000 \mathrm{mg} / \mathrm{m}^{3}\right) \mathrm{IT}=80-89$

10 hipereutrófico $\left(\geq 426.000 \mathrm{mg} / \mathrm{m}^{3}\right)$ IT $=>90$

11 euritrófico, sin preferencia por biotopos ricos o pobres en nutrimentos

Forma de vida

1 béntica

2 perifítica

3 plánctica

Preferencia térmica

1 oligotérmica $\left(-3^{\circ} \mathrm{C}\right)$

2 oligo-mesotérmica

3 meso-oligotérmica

4 mesotérmica $\left(18^{\circ} \mathrm{C} \mathrm{a}-3{ }^{\circ} \mathrm{C}\right)$

5 meso-politérmica $\left(>18{ }^{\circ} \mathrm{C}-35^{\circ} \mathrm{C}\right)$

6 poli-mesotérmica

7 euritérmica (alta temperatura) 
CUADRO 1 (Continuación)

Clasificación de la composición ecológica o valores indicadores

TABLE 1 (Continued)

Classification of ecological composition or indicator values

Florecimientos

1 asociado

2 no masivo ( $\geq 1000$ células $\mathrm{ml}^{-1}$ )

3 masivo ( $\geq 10000$ células $\left.\mathrm{ml}^{-1}\right)$

4 hipermasivo ( $\geq 50000$ células $\mathrm{ml}^{-1}$ )

Frecuencia o distribución

1 muy baja (alrrededor del 10\%)

2 baja (11-30\%)

3 moderada (31-50\%)

4 ligeramente alta $(51-75 \%)$

5 alta $(76-100 \%)$

Dominancia

1 no dominante $<1$

2 dominante $\geq 1$

Intervalos del valor indicador de la calidad del agua

$\leq 1.818$ alta calidad del agua

1.819-3.636 moderada calidad del agua

3.637-5.455 baja calidad del agua

Simbología del potencial de hidrógeno $(\mathrm{pH})$, salinidad $(\mathrm{H})$, suministro metabólico de nitrógeno $(\mathrm{N})$, relación nitrógeno total-fósforo total, saprobiedad (IS), preferencia trófica (Tp), forma de vida (Lf), preferencia térmica (T), florecimientos (B), frecuencia o distribución (F), dominancia (D) e intervalos del valor indicador de la calidad del agua (IVICA) de acuerdo a Caljon (1983), Contreras-Espinosa et al. (1994), Van Dam et al. (1994), Moreno-Ruiz (2000, 2005) y Moreno (2003).

El promedio de los valores indicadores ponderados corresponde al valor indicador de la calidad del agua de los taxones analizados (Apéndice 1), y es el que se compara con los intervalos referidos en el párrafo anterior.

Listado florístico y distribución: para comparar la distribución de las especies presentes en el río Tehuantepec con los estudios realizados en el resto del país, se consultó la información referida por 65 autores desde
1838 hasta 2003 , mismos que se mencionan en Moreno-Ruiz (2005). Con la adición de nueva información a través del análisis de materiales recolectados por los autores del presente trabajo en los 31 estados y localidades de la República mexicana. El material fósil pertenece al Mioceno tardío (Moreno-Ruiz y Carreño 1994), con su recolección por parte de personal del Instituto de Geología de la UNAM y del primer autor. La ilustración y morfometría de las especies sobresalientes se presentan en las 
obras de Moreno-Ruiz (2000 y 2005). Con la intención de conocer parte de la estructura de las comunidades fitopláncticas, se aplicó el índice: riqueza de especies (N0) referido por Moreno (2003).

Para el análisis de las algas microscópicas del río Tehuantepec se estructuró una matriz con el valor indicador ponderado de cada taxon (Apéndice 1), mismo que se estandarizó de acuerdo con Lepistö y Rosenström (1998). En el análisis biogeográfico, se construyó otra matriz binaria de presencia y ausencia de los taxones compartidos entre el río y los del resto del país. Con ambos datos, se clasificaron las estaciones de muestreo, los estados y localidades del país a través del agrupamiento de mínima variancia (método de Ward 1963) y su corrimiento se realizó con el paquete estadístico "STATISTICA" v 5.1 de StatSoft Inc.

\section{RESULTADOS}

Listado florístico: de acuerdo con la composición micoalgal, se registraron 298 taxones, pertenecientes a siete divisiones, 58 familias, un incertae sedis, 121 géneros, 273 especies, una subespecie, 75 variedades, 13 formas y un morfotipo (Apéndice 1).

La División Bacillariophyta sobresalió con 125 taxones (42.0\%), Chlorophyta tuvo 88 taxones $(29.0 \%)$, Cyanoprocaryota exhibió 53 (18.0\%), Euglenophyta mostró 14 (5.0\%), Dinophyta tuvo nueve (3.0\%), Cryptophyta exhibió cinco $(2.0 \%)$ y Chrysophyta cuatro $(1.0 \%)$.

Las familias mejor representadas fueron Scenedesmaceae (24 especies), Oocystaceae (22), Bacillariophyceae (21), Chaetocerotaceae (15) y Euglenaceae (14) quienes mostraron una considerable riqueza de especies.

Comunidades: el fitoplancton exhibió una alta riqueza de especies (157) y estuvo dominado por las cianoprocariotas: Aphanizomenon flos-aquae, Chroococcus minutus, Microcystis flos-aquae y Pseudanabaena limnetica; las diatomeas: Aulacoseira granulata var. granulata f. granulata, Cyclotella meneghiniana y Cyclotella stelligera. Asimismo, se registró en el plancton una menor riqueza de especies perifíticas (26), con la dominancia de Leptolyngbya tenuis (Apéndice 1).

En conjunto con las algas anteriores y de acuerdo al criterio de Caljon (1983), se reconocieron microalgas pertenecientes a la comunidad béntica. Este grupo presentó una baja riqueza de especies (62), y en él, sobresalieron Chroococcus palidus y Peridiniopsis polonicum (Apéndice 1).

De manera similar, entre los vegetales del plancton se registró un grupo de algas microscópicas cuya forma de vida aún no es clara, que mostró una baja riqueza de especies (28), entre las que destacaron Anabaenopsis circularisGymnodinium cf. pasheri (Apéndice 1).

Biogeografía: de las 58 familias (100\%) determinadas en el río Tehuantepec sobresalieron cinco de ellas (8.6\%); entre las restantes categorías sistemáticas resaltaron ocho géneros (6.6\%), 72 especies $(26.4 \%), 45$ variedades (60\%) y ocho formas $(61.5 \%)$ todos estos taxones considerados como nuevos registros para México (Apéndice 1).

La clasificación de las cinco estaciones de muestreo del río Tehuantepec con los 298 taxones, resaltó dos hábitats; el primero se estructuró con las estaciones 1-4, y el segundo con la estación cinco (Fig. 1). En los dos hábitats, sobresalieron 48 especies $(17.6 \%)$ que tuvieron la mayor distribución (Apéndice 1), seis especies $(2.2 \%)$ se distribuyeron en las estaciones $1-4$, y 65 especies $(23.8 \%)$ se presentaron únicamente en una estación de muestreo.

La similitud de algas microscópicas del río Tehuantepec con respecto a 31 estados y localidades de la República mexicana (Apéndice 1, Fig. 2) (Ehrenberg 1838, Moreno-Ruiz 2000, Siqueiros-Beltrones 2002, Moreno-Ruiz 2005) fue de $91.4 \%$ (familias), $93.4 \%$ (géneros), $73.6 \%$ (especies), $10.1 \%$ (variedades) y $1.7 \%$ (formas). Con la mayor riqueza de bacilariofitas (106 especies), seguidas por las clorofitas (63 especies); así como la menor riqueza de dinofitas (seis especies) y criptofitas (tres especies). 


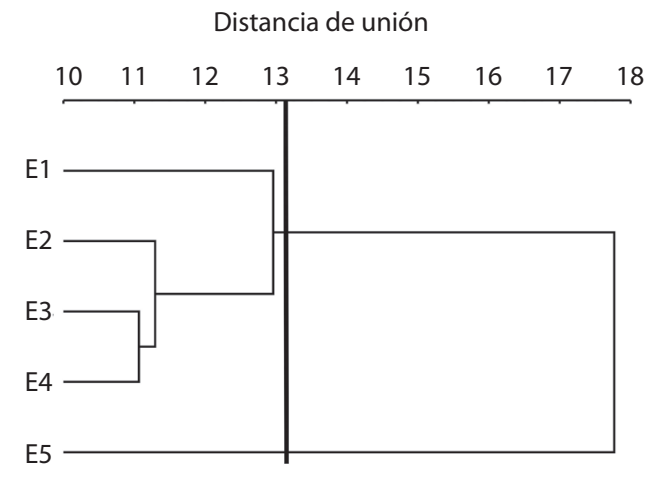

Fig. 1. Dendrograma del análisis de cinco biotopos del río Tehuantepec en cinco periodos y estaciones de muestreo de acuerdo con la composición de 298 taxones de algas microscópicas y valores promedio ponderados de $\mathrm{pH}$, salinidad, suministro metabólico de nitrógeno, relación nitrógeno total:fósforo total, saprobiedad, preferencia trófica, forma de vida, preferencia térmica, florecimientos, frecuencia o distribución y dominancia. La flecha muestra la línea de división arbitraria que definió los dos hábitats.

Fig. 1. Dendrogram for cluster analysis of five biotopes from Tehuantepec River in five periods and sampling sites using taxonomic composition of 298 taxa of microscopic algae and average pondered values of $\mathrm{pH}$, salinity, nitrogen uptake metabolism, Total-N/Total-P ratios, saprobity, trophic preference, life form, thermic preference, blooms, frequency or distribution and dominance. The vertical arrow shows the arbitrary division line for defining two habitats.

La clasificación de los estados y localidades de la República mexicana por medio de los 298 taxones determinados, destacó cinco hábitats (Fig. 2): el primero se formó con seis localidades de las regiones suroccidental a noroccidental en la vertiente del Pacífico mexicano (ChiapasGolfo de California), el segundo por siete estados de la región central occidental a oriental (Guerrero-Puebla), el tercero abarcó 13 estados y localidades de las regiones sur-oriental, subcentral, norcentral y noroccidental (JaliscoMorelos); el cuarto por un estado de la región subcentral suroccidental (Oaxaca), y el quinto a los estados ubicados en las regiones central occidental a nororiental y suroriental (GuerreroMichoacán a Tabasco). En estos cinco hábitats resaltaron tres especies $(1.1 \%)$ con amplia

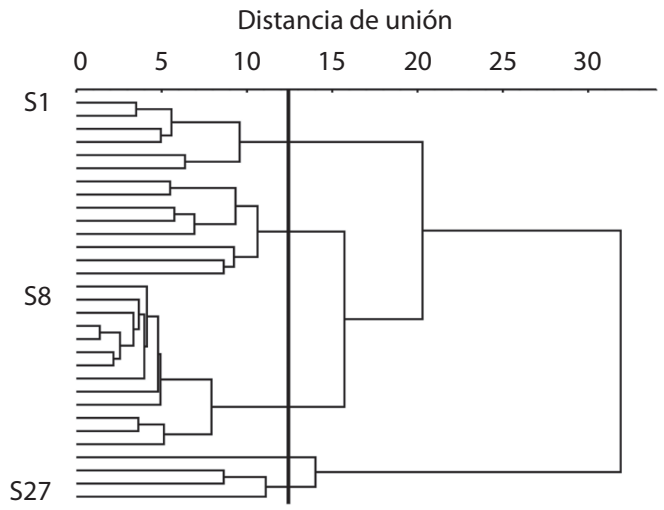

Fig. 2. Dendrograma del análisis de agrupamiento de 31 localidades y estados del país respecto a 298 taxones de algas microscópicas: (S1) Chiapas, (S2) Chiapas-Oaxaca, (S3) Oaxaca, (S4) Guerrero, (S5) Guerrero-Michoacán, (S6) Michoacán, (S7) Colima, (S8) Jalisco, (S9) Nayarit, (S10) Costa occidental (CO) de Baja California Sur, (S11) CO de Baja California Sur-Baja California Norte, (S12) CO de Baja California Norte, (S13) Golfo de California, (S14) Coahuila, (S15) Chihuahua, (S16) Distrito Federal, (S17) Estado de México, (S18) Guanajuato, (S19) Hidalgo, (S20) Morelos, (S21) Nuevo León, (S22) Puebla, (S23) Querétaro, (S24) San Luis Potosí, (S25) Sinaloa, (S26) Sonora, (S27) Tabasco, (S28) Tamaulipas, (S29) Tlaxcala, (S30) Veracruz y (S31) Yucatán. La flecha muestra la línea de división arbitraria que definió los cinco hábitats.

Fig. 2. Dendrogram for cluster analysis of 31 localities and states from Mexico using taxonomic composition of 298 taxa of microscopic algae (see sites above). The vertical arrow shows the arbitrary division line for defining five habitats.

distribución, 40 especies (14.7\%) exhibieron una moderada distribución y 60 especies (22\%) sólo se registraron en un estado.

La comunidad microalgal de suramérica (Yacubson 1974, Parra et al. 1982, González De Infante 1988, Soler 1993, Soler et al. 1993, Vyverman 1996, Huszar et al. 1998, DíazRamos 2000, Donato-Rondón 2001, Riofrío et al. 2003, Lacerda et al. 2004) tuvo la similitud con las algas microscópicas del río Tehuantepec (Apéndice 1) de $79.3 \%$ (familias), $56.2 \%$ (géneros), $41 \%$ (especies), 46.7 $\%$ (variedades) y $53.8 \%$ (formas). Entre estas algas, las bacilariofitas exhibieron una gran 
riqueza de especies (60) y en segundo lugar las clorofitas (30 especies); con la menor riqueza concentrada entre las euglenofitas (dos especies) y criptofitas (una especie).

\section{DISCUSIÓN}

Para el río Tehuantepec, se tiene el registro de 298 taxones de algas microscópicas, en virtud de que es el primer estudio que se realiza en esta área, entre ellas, sobresalieron las especies fitopláncticas $(57.5 \%)$ sobre las perifíticas $(9.5$ $\%$ ) y bénticas $(22.7 \%)$. Sin embargo, resaltó un menor número de especies cuya forma de vida fue incierta $(10.3 \%)$ y que pudo ser el resultado de los métodos de muestreo que fueron específicos para recolectar algas del plancton. Por lo que se requieren incorporar otras técnicas para tener una ubicación más precisa de todos los componentes microalgales y por ende de las comunidades y subcomunidades referidas por Round (1981).

Con base en la composición microalgal, fue posible caracterizar a un tramo del río, como un hábitat acuático limnético (estaciones 1-4); a otro (estación cinco) con fluctuación limnética (agua dulce con salinidad $<0.5 \%$ ) a oligohalina, con los taxones característicos Actinastrum hantzschii var. hantzschii que es limnobionte débilmente eurihalino (habita en agua dulce, pero tolera condiciones oligohalinas 0.5-3 \%o), Cylindrotheca closterium y Entomoneis alata que tipifican condiciones salobres eurihalinas (se desarrollan entre 2-20 $\%$, Caljon 1983). Asimismo, se distinguieron oscilaciones marinas eurihalinas en particular, por la presencia de las especies de los géneros Asterionellopsis, Bacteriastrum, Chaetoceros, Proboscia, Pseudo-nitzschia, Rhizosolenia y Thalassionema.

En la zona costera, las lagunas costeras adyacentes y en la zona marina, se han realizado algunos trabajos fitopláncticos de índole taxonómico y ecológico en los que se trataron especies particularmente marinas (Tapia-García et al. 1998). Sin embargo, al comparar y confirmar la presencia de varias especies pláncticas, en la estación cinco aledaña al mar, se registraron células solitarias de Chaetoceros subtilis var. subtilis f. knipowitzschii y de C. seiracanthus. Este encuentro fue interesante, ya que solo en condiciones ambientales particulares o en cultivo diversas especies de Chaetoceros tienden a formar unicélulas (Rines y Hargraves 1988) y en estas dos especies, hasta donde los autores tienen conocimiento, no se habían observado, por lo que es probable que estas variaciones morfológicas pudieron resultar de las oscilaciones de la salinidad.

En la clorofita Spermatozopsis, Caljon (1983) separó a Spermatozopsis sp. 1 de $S$. exultans porque presentó dos flagelos (undulipodios). Aunque probablemente fue una separación parcial, ya que la especie nominal se caracteriza por tener generalmente cuatro y en ocasiones dos undulipodios. En el río Tehuantepec se observó la misma situación, pero a la vez, destacaron ejemplares sin undulipodios en la estación cinco (hábitat de alta tensión, por la mezcla del agua dulce y marina que se efectúa aquí), lo que evitó su asignación en taxones separados, ya que entre otros parámetros, las fluctuaciones de la salinidad pudieron ser las responsables de esa variación morfológica (Moreno-Ruiz 2005), pero se requiere cultivar a esta especie para tener una delimitación sistemática más clara e incrementar el conocimiento de su composición ecológica.

De manera similar sobresalieron las euglenofitas y criptofitas entre las estaciones de muestreo 1-4, como probable respuesta a considerables cantidades de materia orgánica, derivadas de las actividades urbanas, debido a que el río atraviesa los poblados de Santa María Mixtequilla, Santo Domingo, Tehuantepec y San Blas Atempa, donde se le utiliza para descargas de aguas negras, tiradero de basura y otros desechos domésticos (Tapia-García et al. 1998).

Destacaron las especies de amplia distribución Chroococcus turgidus, M. flos-aquae y P. limnetica, así como Chroococcus dispersus, Marssoniella elegans y Rhabdoderma lineare, que por su permanencia y abundancia, repercutieron en la dinámica ecológica 
que se desarrolla en el río, ya que al tratarse de especies con células pequeñas altamente competitivas, pudieron excluir a las especies con células más grandes (Oviat et al. 1989) en ambientes con cierta estratificación (Nielsen y Kiørboe 1991) entre las estaciones 1-4. En contraste, la estación cinco mostró eventos de turbulencia, que se detectan por el descenso de la abundancia de las cianoprocariotas anteriormente referidas, y del desarrollo de las especies grandes: Chaetoceros spp., Cerataulina pelagica, Guinardia striata, Proboscia alata $\mathrm{f}$ alata, Rhizosolenia spp., Bacillaria paxillifer y de otras especies fitopláncticas y fitobénticas (Moreno-Ruiz 2005), por lo que fue más evidente el proceso de mezcla asociado a los movimientos de marea y a los "tehuantepecanos", que generaron un hábitat de características estuarinas.

Las especies de Chaetoceros y Thalassionema al caracterizar surgencias (Sancetta 1983, Marshall y Cohn 1987), evidenciaron este proceso en la estación cinco, aunado al incremento de la salinidad, enriquecimiento de nutrimentos e incremento de la productividad, que posiblemente resultaron de la surgencia costera postulada para la parte norte del Golfo de Tehuantepec (Roden 1961, Stumpf 1975).

Con base en la similitud y clasificación por conglomerados (Fig. 1), el hábitat estructurado por las estaciones 1-4 resaltó por la exclusiva distribución de los seis taxones: M. elegans, Cephalomonas granulata, Gomphonema affi$n e, R$. lineare, Cryptomonas ovata var. ovata, Cryptomonas ovata var. curvata y Sellaphora pupula var. capitata, especies limnobiontes a limnobiontes eurihalinas de primer grado, más una baja riqueza de especies. De manera opuesta, el hábitat constituido por la estación cinco se diferenció por 65 especies únicas: entre las que sobresalió el componente limnético Arthrospira spirulinoides-Pinnularia cardinalis, así como el componente marino Amphora terroris-Thalassionema frauenfeldii en el área de mayor riqueza de especies. Sin embargo, entre ambos hábitats aunados a las características ambientales resumidas en el valor de importancia ponderado, exhibieron la moderada calidad del agua resultante de las actividades urbanas, ganaderas e industriales que aquí se desarrollan (TapiaGarcía et al. 1998).

La clasificación de los estados del país (Fig. 2), mostró la mayor distribución de los tres taxones limnobiontes eurihalinos: A. granulata var. granulata f. granulata, C. meneghiniana y Synedra ulna var. ulna. De los cinco hábitats, el primero se definió por una moderada riqueza de taxones (28-77) y compartió 28 especies entre las que sobresalieron Nitzschia sigmaRhizosolenia clevei var. comunis que oscilaron entre una composición salobre eurihalina superior a marina eurihalina. El segundo hábitat exhibió de manera similar una moderada riqueza de taxones (26-74), pero destacaron por su distribución las tres especies limnobiontes eurihalinas de primer grado: Chlorella vulgaris, Tetraedron minimum y Caloneis amphisbaena. El tercer hábitat mostró la menor riqueza de especies (1-24) y en él resaltó por su distribución la especie limnobionte eurihalina de primer grado Gomphonema gracile var. gracile. El cuarto hábitat tuvo la mayor riqueza de especies (298) y en él sobresalieron los 58 taxones limnobiontes y limnobiontes eurihalinos: Anacystis nidulans-Peridiniopsis cunningtonii. El quinto hábitat exhibió una alta riqueza de taxones (103-140), en el que resaltaron por su distribución 16 especies cuya composición ecológica fluctuó de limnobionte a limnobionte eurihalina entre Anabaena cf. cylindrica a $P$. limnetica (Apéndice 1).

Banderas-Tarabay (1997) refirió la similitud de especies entre su estudio y la República mexicana del $40.4 \%$ al considerar únicamente la distribución referida por Ortega (1984). Opuestamente en este trabajo al consultar a más autores (Moreno-Ruiz 2005) y por análisis propios, se obtuvo el incremento del $83.9 \%$ de especies compartidas. Sin embargo, de los cinco hábitats, el tercero está prácticamente inexplorado y se requieren realizar más investigaciones de la composición abundancia y distribución de las algas microscópicas en el país para establecer una mayor relación florística.

En el ámbito tropical, la comparación de la flora microalgal suramericana con la mexicana, 
exhibió a la especie limnobionte eurihalina $A$. granulata var. granulata f. granulata con una distribución ligeramente alta. Otras ocho especies limnobiontes eurihalinas: Ankistrodesmus falcatus var. falcatus-Synedra ulna var. ulna, más la especie holeurihalina $B$. paxillifer tuvieron una distribución moderada. 33 especies entre limnobiontes como Pediastrum simplex var. simplex a la salobre eurihalina superior E. alata mostraron una distribución baja, y 76 especies con representantes limnobiontes como Achnanthes exigua var. heterovalvata y la especie marina eurihalina Thalassionema nitzschioides var. nitzschioides exhibieron una distribución muy baja (Apéndice 1).

Al considerar la hipótesis de un origen común y amplia dispersión de la flora algal desde Suramérica a Norteamérica, BanderasTarabay (1997) registró la similitud de especies del $14 \%$. Sin embargo, aunque en el presente estudio se obtuvo el $41 \%$, esta distribución mostró aún la existencia de localidades pobremente estudiadas en Suramérica y México, por lo que la implementación de más estudios, tendrá probablemente como resultado el incremento del número de especies compartidas y en consecuencia una mayor correspondencia microalgal.

\section{AGRADECIMIENTOS}

El presente estudio forma parte de las investigaciones de posgrado del primer y cuarto autores, por lo que se expresa especial agradecimiento a la Universidad Autónoma Metropolitana, CONACyT, Instituto Mexicano del Petróleo, DGAPA, Universidad Nacional Autónoma de México (UNAM) y a la SEP (FOMES) por los apoyos proporcionados. A Margarita Reyes y Jorge Sepulveda del Instituto de Geología e Instituto de Fisiología Celular, UNAM, respectivamente por su ayuda con microscopía electrónica. A Susan Carty de Texas A \& M por sus valiosos comentarios que enriquecieron el análisis de la composición, en particular de las dinoflageladas, y a Herman van Dam de Aqua Sence TEC,
Amsterdam, por sus comentarios sobre la composición ecológica.

\section{RESUMEN}

El fitoplancton recolectado en el río Tehuantepec con características limnéticas a eurihalinas se determinó, clasificó y comparó con información regional de México y Suramérica. Se incluyen valores indicadores y un código del listado florístico para facilitar el manejo de estas algas. Se determinó un total de 58 familias, 121 géneros, 273 especies, una subespecie, 75 variedades, 13 formas y un morfotipo, pertenecientes a las divisiones Bacillariophyta (42.0\%), Chlorophyta (29.0\%), Cyanoprocaryota (18.0\%), Euglenophyta (5.0\%), Dinophyta (3.0\%), Cryptophyta $(2.0 \%)$ y Chrysophyta $(1.0 \%)$. Las familias mejor representadas fueron Scenedesmaceae (24 especies), Oocystaceae (22), Bacillariophyceae (21), Chaetocerotaceae (15) y Euglenaceae (14). Se establecen nuevos registros para México: cinco familias, ocho géneros, 72 especies, 45 variedades y ocho formas. En este río, que presenta una moderada calidad del agua, sobresalieron las especies Chroococcus turgidus, Microcystis flosaquae y Pseudanabaena limnetica (como productoras de mareas rojas).

Palabras clave: fitoplancton, neotropical, limnético, eurihalino, valores indicadores, florecimientos masivos, calidad del agua.

\section{REFERENCIAS}

Anónimo. 1981. Carta topográfica Juchitán número 15-10, D15-1. Instituto Nacional de Estadística Geografía e Informática (INEGI), Esc. 1:250 000, México D.F., México.

Anónimo. 2000. Estudio ambiental integral de la región de Salina Cruz, Oaxaca, México, Río Tehuantepec, Marzo 2000. Instituto Mexicano del Petróleo (IMP), México D.F., México.

Banderas-Tarabay, A. 1997. Phycoflora of the tropical high-mountain lake El Sol, Central Mexico, and some biogeographical relationships. Hydrobiologia 354: 17-40.

Caljon, A. 1983. Brackish-water phytoplankton of the Flemish lowland. Dev. Hidrobiol. 18: 1-272.

Contreras-Espinosa, F., O. Castañeda-López \& A. GarcíaNagaya. 1994. La clorofila $a$ como base para un índice trófico en lagunas costeras mexicanas. An. Inst. Cienc. Mar Limnol. Univ. Nal. Autón. México 21: 55-66. 
Díaz-Ramos, J.R. 2000. Índice de la microflora marina de Venezuela: diatomeas, dinoflagelados y cocolitofóridos. Rev. Biol. Trop. 48: 897-918.

Donato-Rondón, J.C. 2001. Fitoplancton de los Lagos Andinos del Norte de Sudamérica (Colombia) Composición y Factores de Distribución. Acad. Col. Cienc. Exactas Fís. Nat., Bogotá, Colombia.

Ehrenberg, C.G. 1838. Die Infusionsthierchen als vollkommene Organisme. Ein Blick in das tiefere organische Leben der Natur. Leopold, Leipzig, Alemania.

García, E. 1981. Modificaciones al sistema de clasificación climática de Copen (para adaptarlo a las condiciones de la República Mexicana). Larios, México D.F., México.

González De Infante, A. 1988. El Plancton de las Aguas Continentales. OEA, Washingto DCn, EEUU.

Huszar, V.L.M., L.H.S. Silva, P. Domingos, M. Marinho \& S. Melo. 1998. Phytoplankton species composition is more sensitive than OECD criteria to the trophic status of three Brazilian tropical lakes. Hidrobiologia 369/370: 59-71.

Lacerda, S.R., M.L. Koening, O.S. Neuman-Leitã \& M.J. Flores-Montes. 2004. Phytoplankton nyctemeral variation at a tropical river estuary (ItamaracáPernambuco-Brazil). Braz. J. Biol. 64: 81-94.

Lepistö, L. \& U. Rosenström. 1998. The most typical phytoplankton taxa in four types of boreal lakes. Hydrobiologia 369/370: 89-97.

Marshall, H.G. \& M. Cohn. 1987. Phytoplankton composition of the New York Bight and adjacent waters. J. Plank. Res. 9: 267-276.

Monreal-Gómez, M.A. \& D.A. Salas De León. 1998. Dinámica y estructura termohalina, p. 13-26. In M. Tapia (ed.). El Golfo de Tehuantepec: el ecosistema y sus recursos. Univ. Autón. Metropol., Itzapalapa, México.

Moreno, J.L. 2003. Fitoplancton, p. 13-45. In G. De La Lanza-Espino \& S. Hernández-Pulido (eds.). Manual para la recolecta, el manejo y las observaciones de campo para bioindicadores de calidad del agua. CNA/ AGT, México D.F., México.

Moreno, J.L., S. Licea \& H. Santoyo. 1996. Diatomeas del Golfo de California. Universidad Autónoma de Baja California Sur, SEP-FOMES/PROMARCO, Baja California Sur, México.
Moreno-Ruiz, J.L. 2000. Fitoplancton, p. 43-108. In G. De La Lanza Espino, S. Hernández-Pulido \& J.L. Carbajal-Pérez (eds.). Organismos indicadores de la calidad del agua y de la contaminación. SEMARNAP, CNA, UNAM, Inst. Biol. UNAM, Plaza \& Valdez, México D.F., México.

Moreno-Ruiz, J.L. 2005. Fitoplancton, p. 33-64. In J.S. Bueno-Soria \& F. Álvarez-Noguera (eds.). Biodiversidad de Tabasco. Instituto de biología, UNAM/CONABIO, México D.F., México.

Moreno-Ruiz, J.L. \& A.L. Carreño. 1994. Diatom biostratigraphy of Bahía Asunción, Baja California Sur, México. Rev. Mex. Cienc. Geol. 11: 243-252.

Nielsen, J.G. \& T. Kiørboe. 1991. Effects of a storm event on the structure of the pelagic food web with special emphasis on planktonic ciliates. J. Plankton Res. 13: 35-51.

Ortega, M.M. 1984. Catálogo de Algas Continentales Recientes de México.UNAM, México DF, México.

Ortega, M.M., J.L. Godinez, G. Garduño-Solorzano \& M.G. Oliva. 1995. Ficología de México: Algas Continentales. AGT, México D.F., México.

Oviat, C., P. Lane, I.F. French \& P. Donaghay. 1989. Phytoplankton species and abundance in response to eutrophication in coastal marine mesocosms. J. Plankton Res. 11: 1223-1244.

Parra, O.O., M. González, V. Dellarossa, P. Rivera \& M. Orellana. 1982. Manual Taxonómico del Fitoplancton de Aguas Continentales, con Especial Referencia al Fitoplancton de Chile. I- Cyanophyceae. Universidad de Concepción, Concepción, Chile.

Rines, J.E.B. \& P.E. Hargraves. 1988. The Chaetoceros Ehrenberg (Bacillariophyceae) flora of Narragansett Bay, Rhode Island, USA. Bibl. Phycol. 79: 1-196.

Riofrío, J., I. Samanez, F. Carrasco \& M. Clavo. 2003. Limnological characteristics of the Amazonic Cashibococha Lake (Ucayali-Peru) at 2001. Rev. Peru Biol. 10: 183-194.

Roden, G.I. 1961. Sobre la circulación producida por el viento en el Golfo de Tehuantepec y sus efectos sobre las temperaturas superficiales. Geofis. Inter. 3: 55-72.

Round, F.E. 1981. The ecology of algae. Cambridge, Cambridge, Inglaterra.

Rzedowsky, J. 1978. La Vegetación de México. Limusa, México D.F., México. 
Sancetta, C.A. 1983. Diatoms in sediments as indicators of the shelf-slope break. Soc. Econ. Pal. Min. Spec. Pub. 33: 373-380.

Siqueiros-Beltrones, D.A. 2002. Diatomeas bentónicas de la Península de Baja California; diversidad y potencial ecológico. CICIMAR, Libros de Ciencias Marinas, IPN-UABCS, La Paz, México.

Soler, A. 1993. Contribuciones al conocimiento de las diatomeas (Bacillariophyceae) de Panamá. II. Epifitismo en Hydrilla verticillata. Rev. Universidad 48: 106-168.

Soler, A., M.Y. Castillo \& E.V. Robles. 1993. Contribuciones al conocimiento de las diatomeas (Bacillariophyceae) de Panamá. I. Diatomeas del Lago Gatún. Rev. Universidad 47: 161-227.

Stumpf, H.G. 1975. Satelite detection of upwelling in the Gulf of Tehuantepec, Mexico. J. Phys. Oceanogr. 5: 383-388.
Tapia-García, M., E. Ramos-Santiago \& A. Ayala-Cortés. 1998. La actividad humana y su impacto en la zona costera con énfasis en el Istmo de Tehuantepec, p. 209-228. In M. Tapia (ed.). El Golfo de Tehuantepec: el ecosistema y sus recursos. Univ. Autón. Metropol., México D.F., México.

Van Dam, H., A. Mertens \& J. Sinkeldam. 1994. A coded checklist and ecological indicador values of freshwater diatoms from the Netherlands. Neth. J. Aquatic Ecol. 28: 117-133.

Vyverman, W. 1996. The Indo-Malaysian North-Australian phycogeographical region revised. Hydrobiologia 336: 107-120.

Ward, J. 1963. Hierarchical grouping to optimise an objetive function. J. Amer. Statist. Ass. 58: 236-244.

Yacubson, S. 1974. Catálogo e iconografía de las Chlorophyta de Venezuela. Bol. Centro Invest. Biol. Univ. Zulia, Fac. Hum. Educ. Maracaibo, Venezuela. 


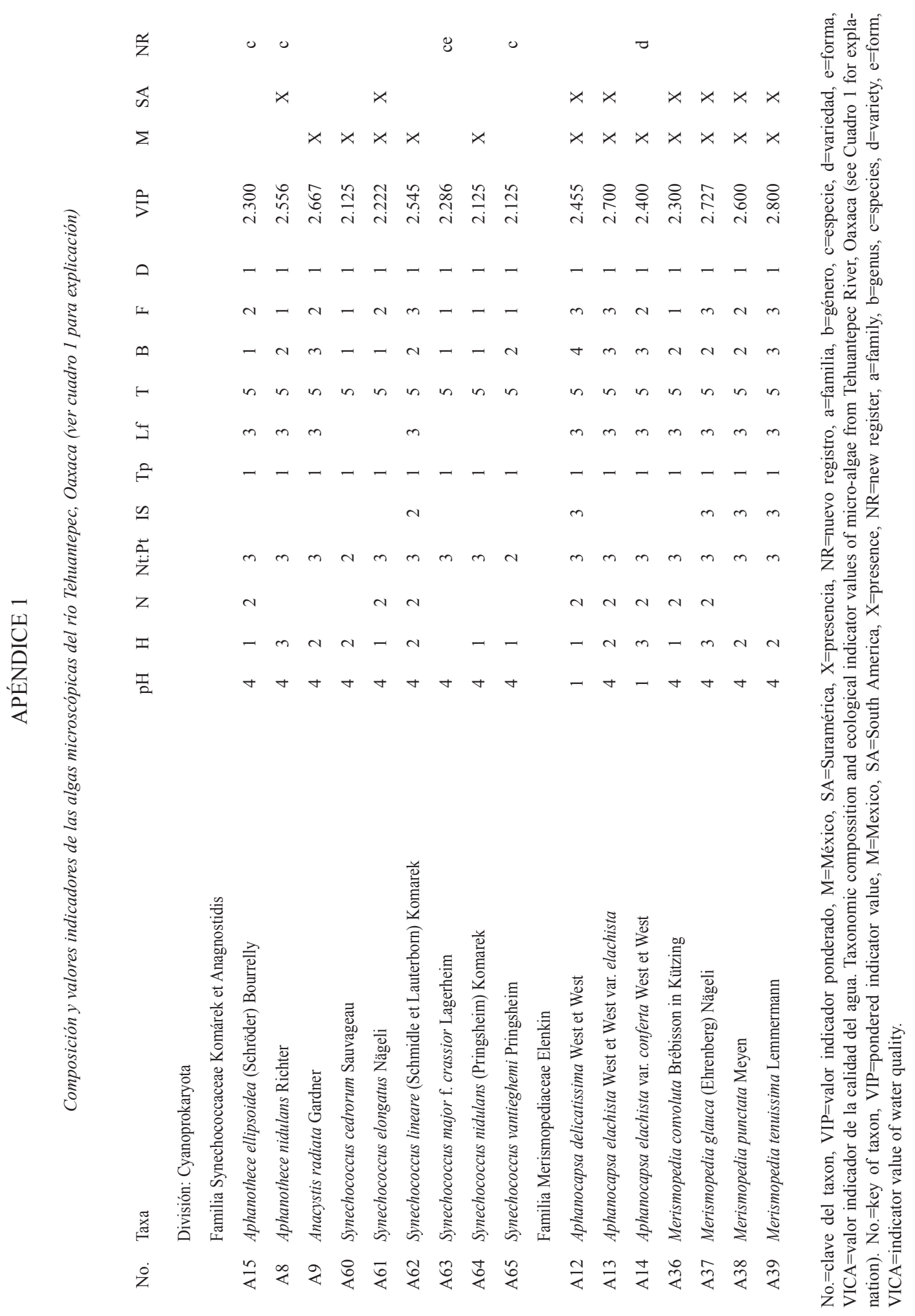




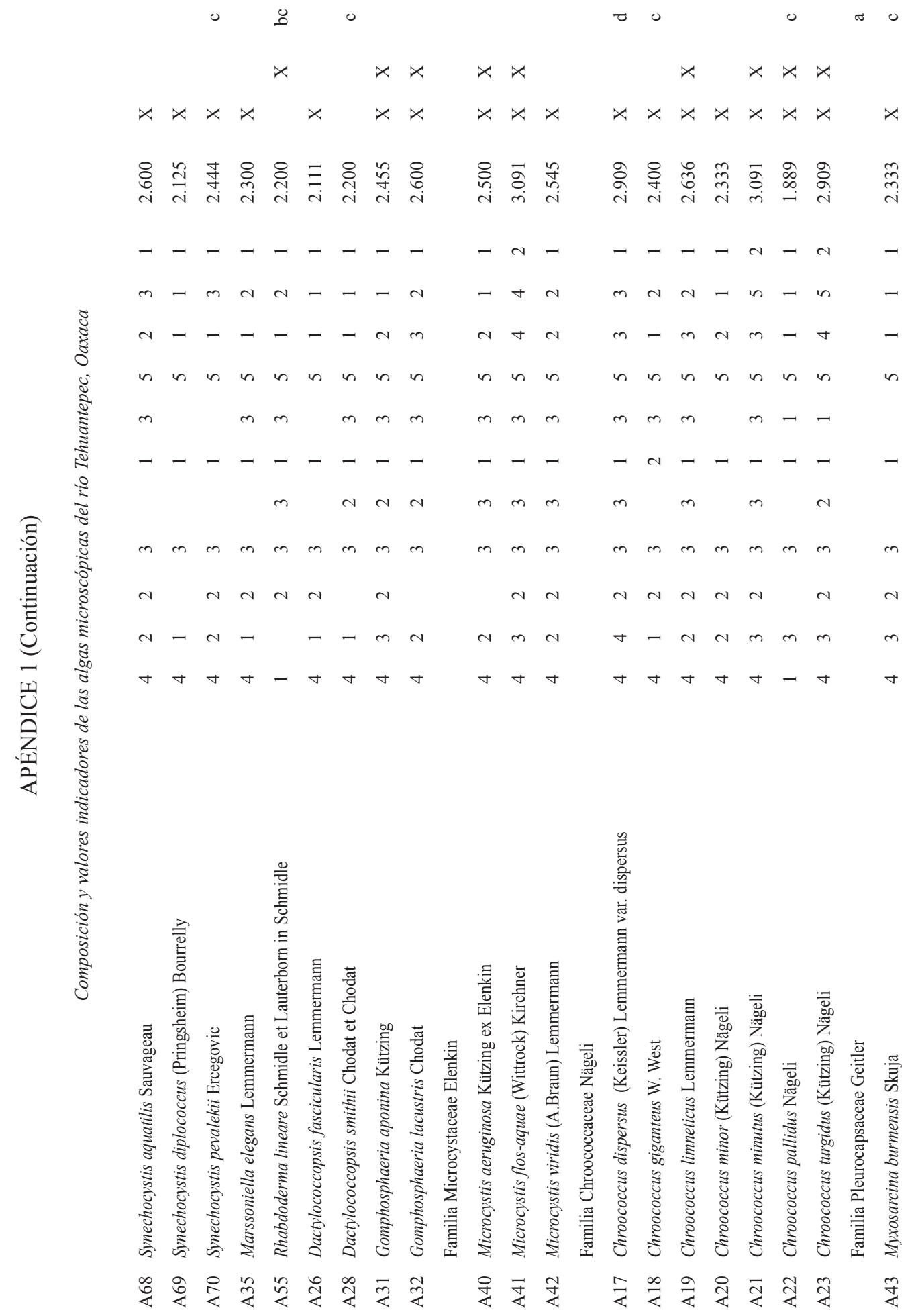




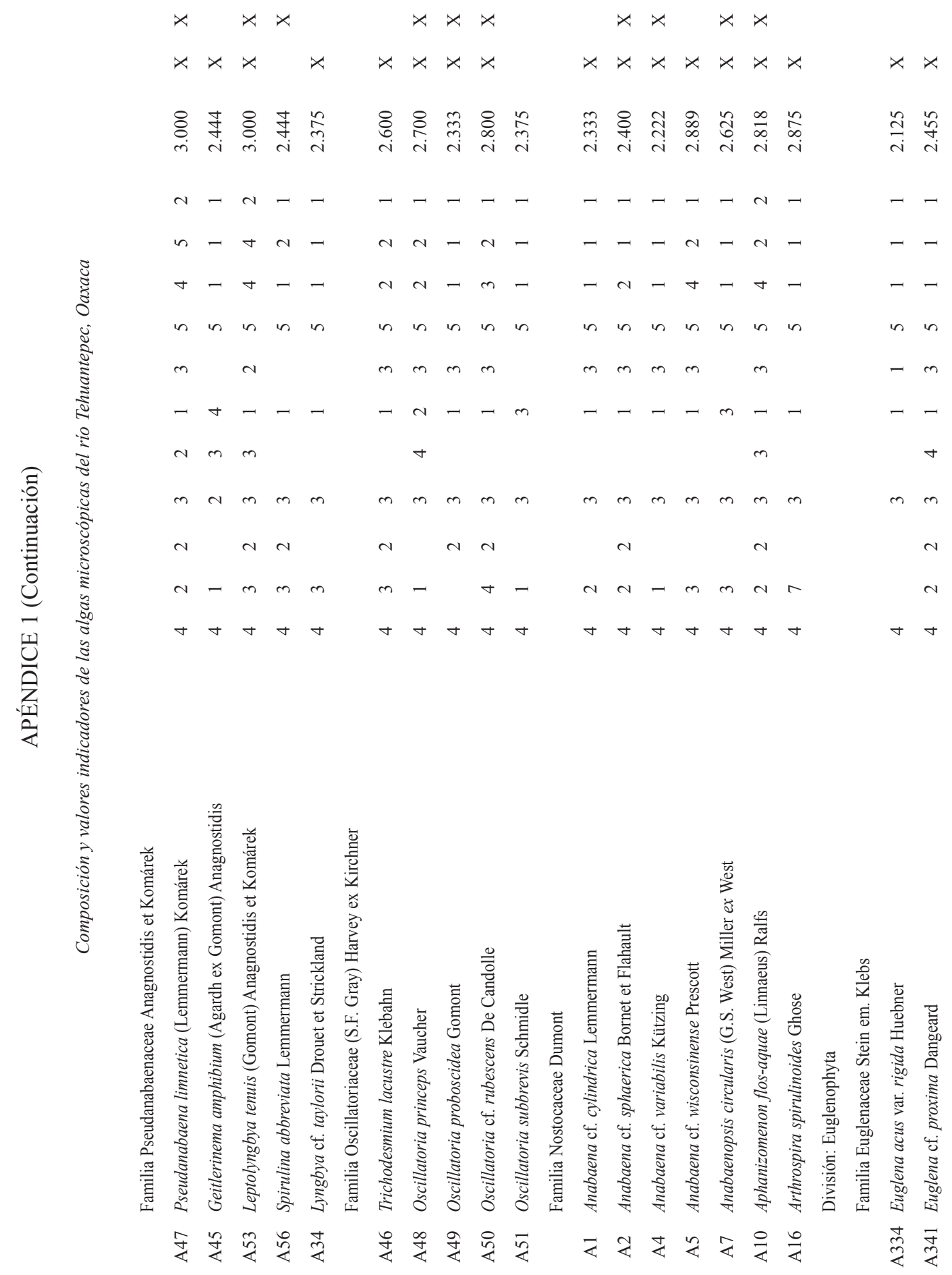




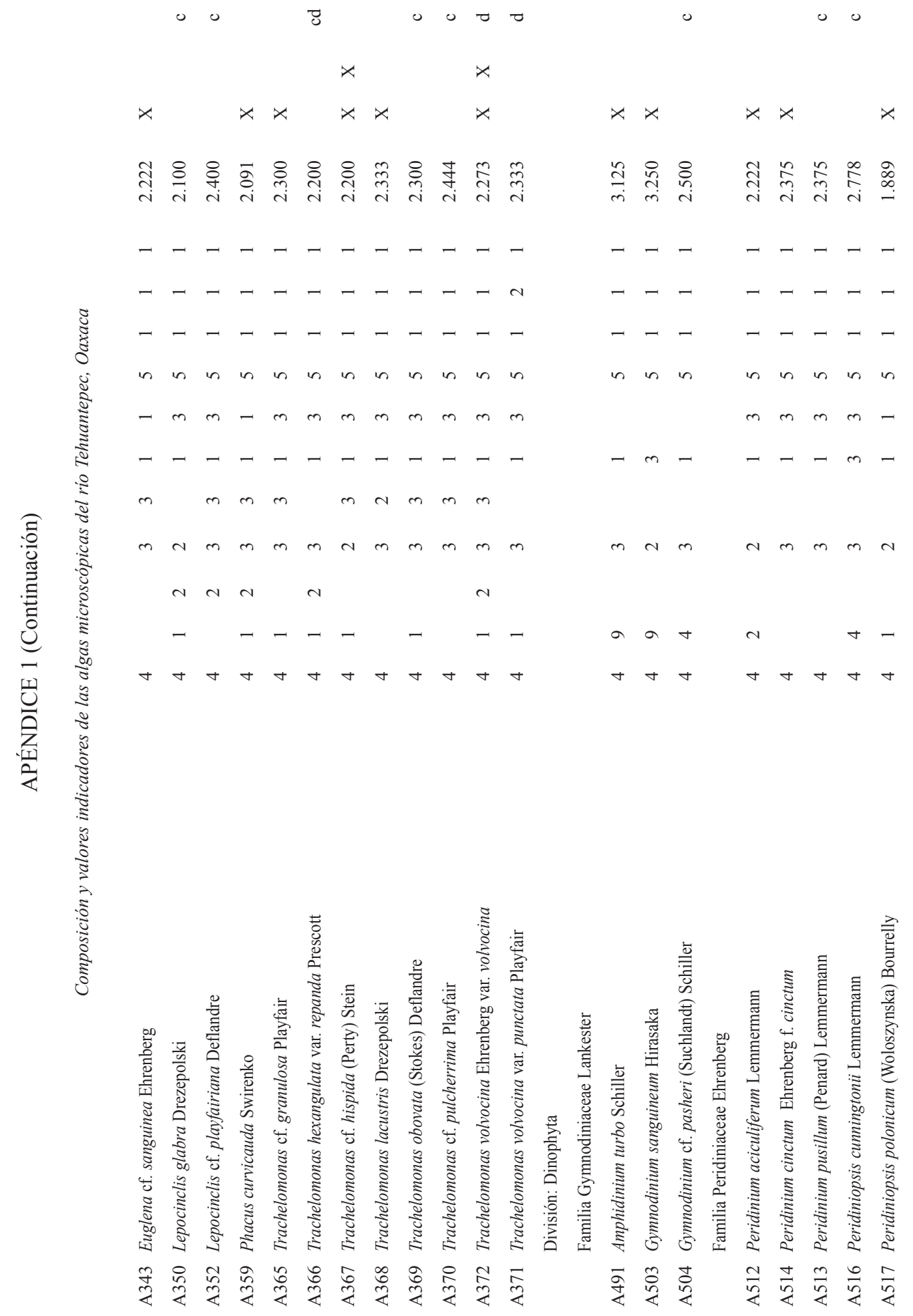




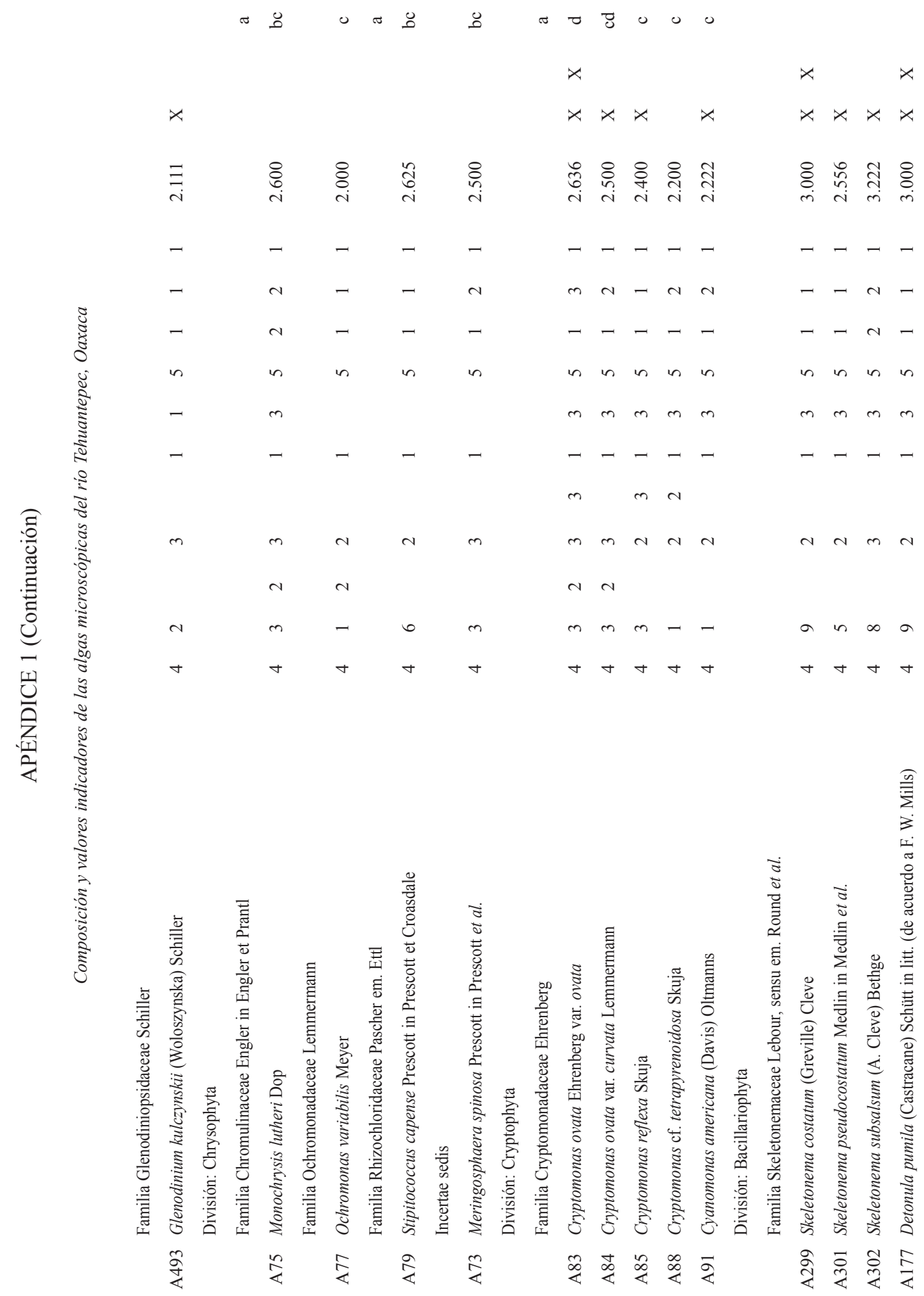



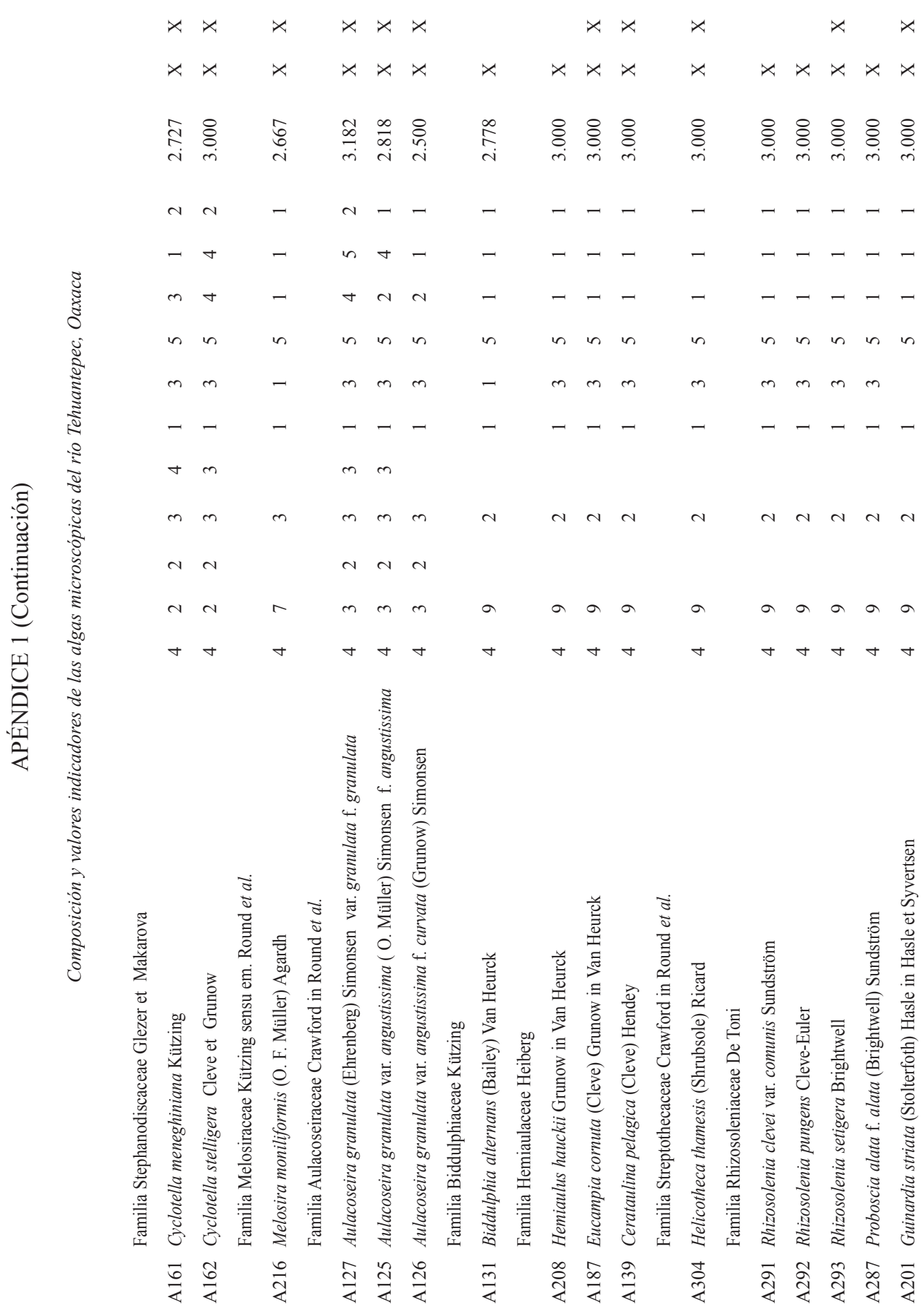

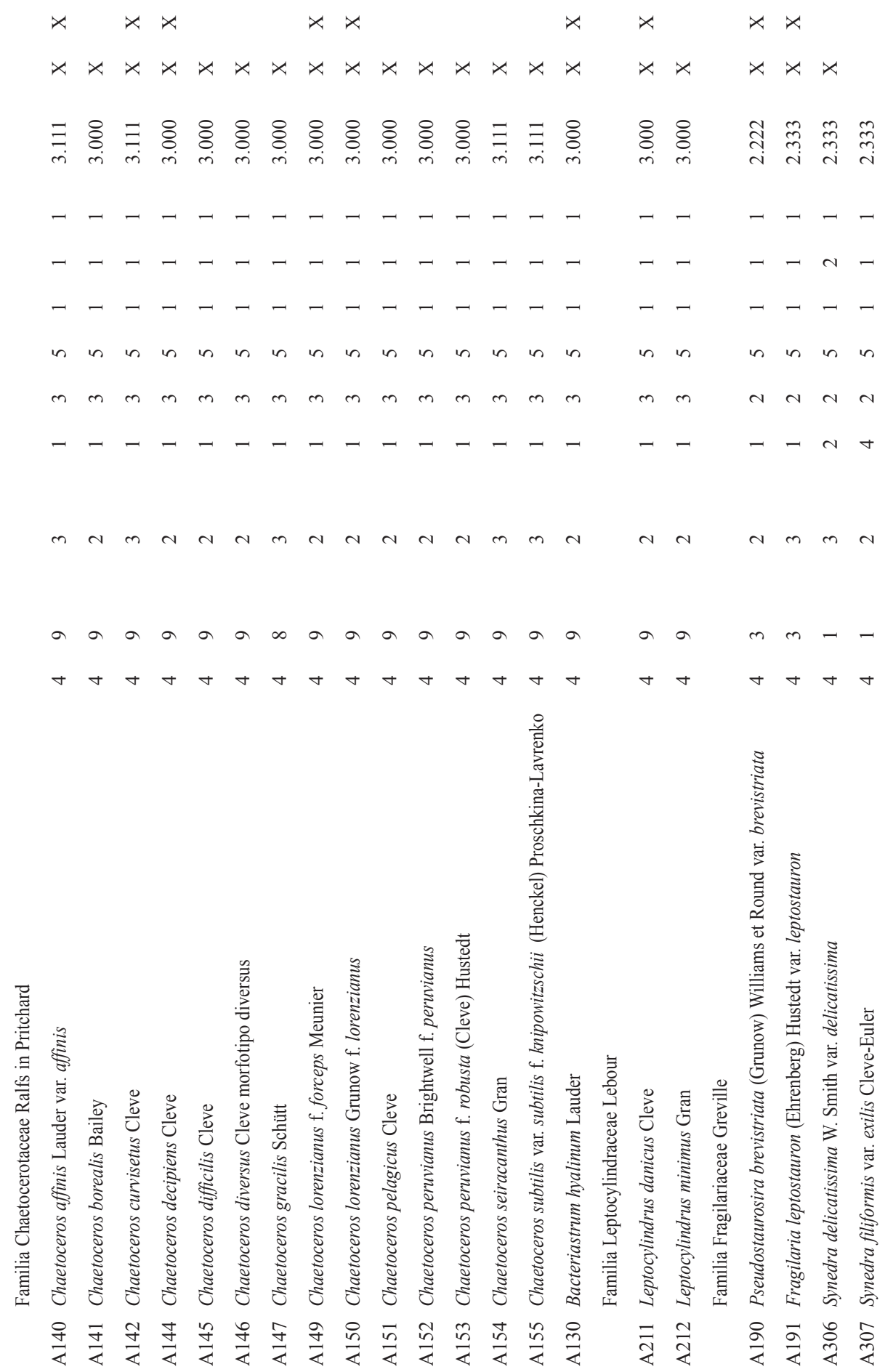


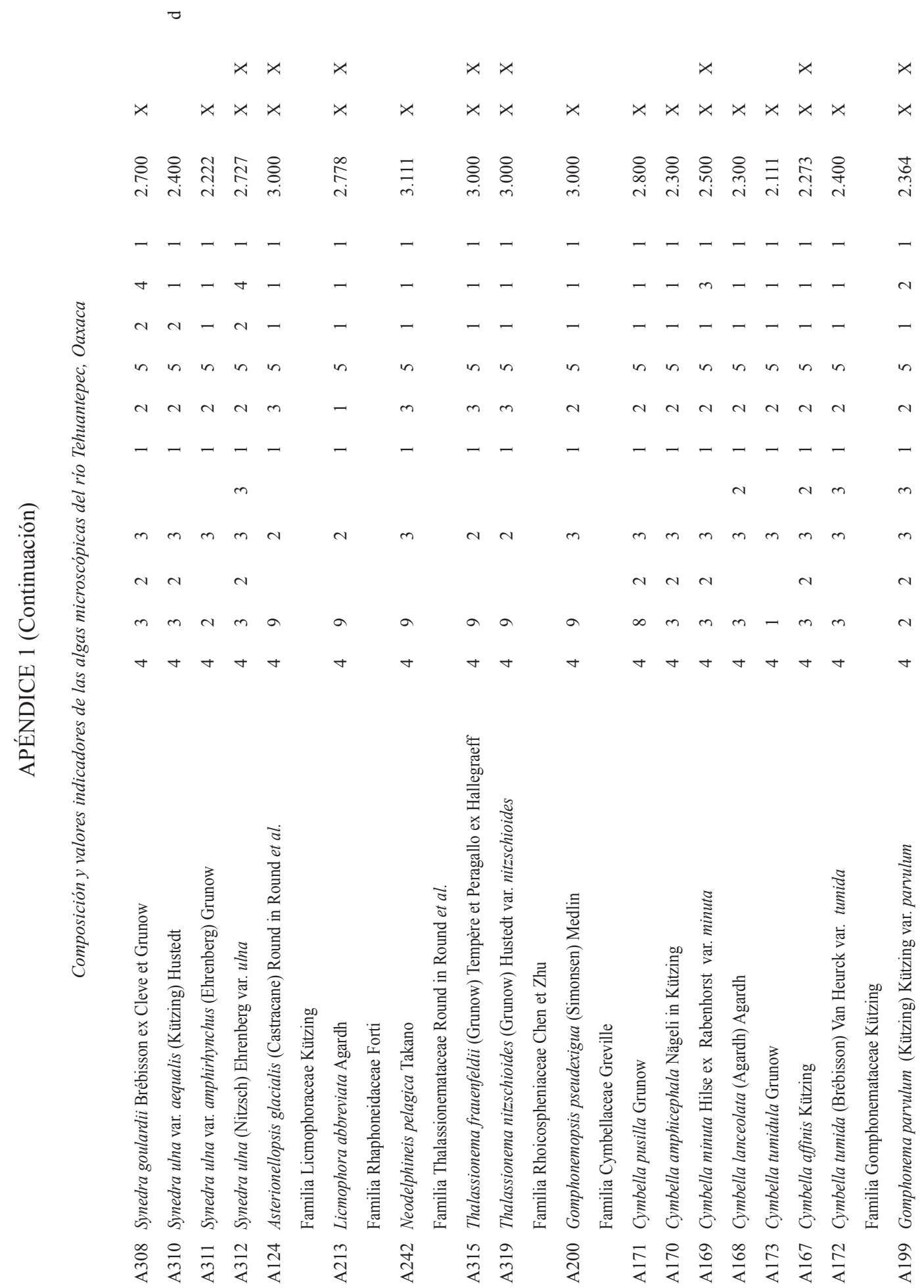




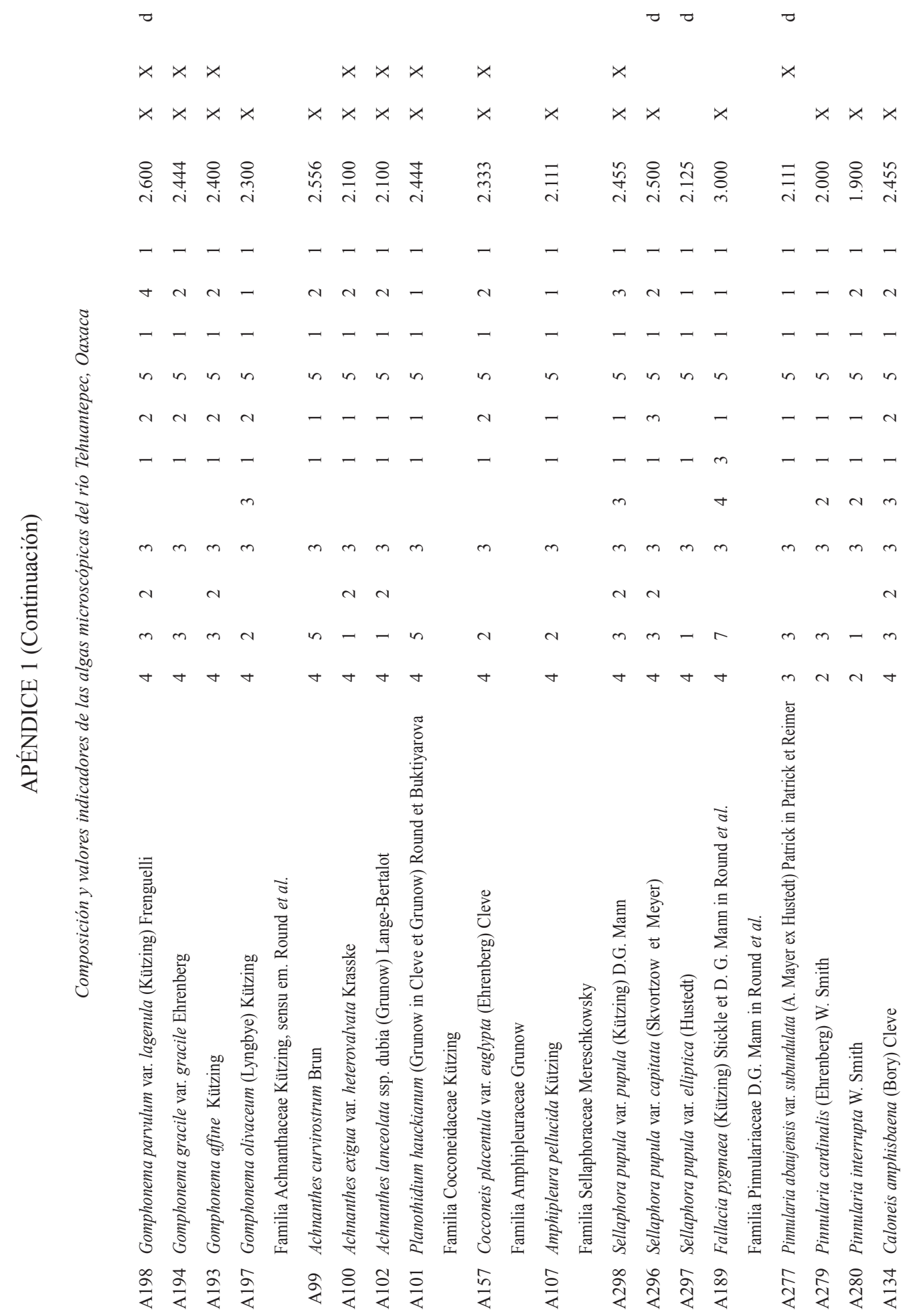




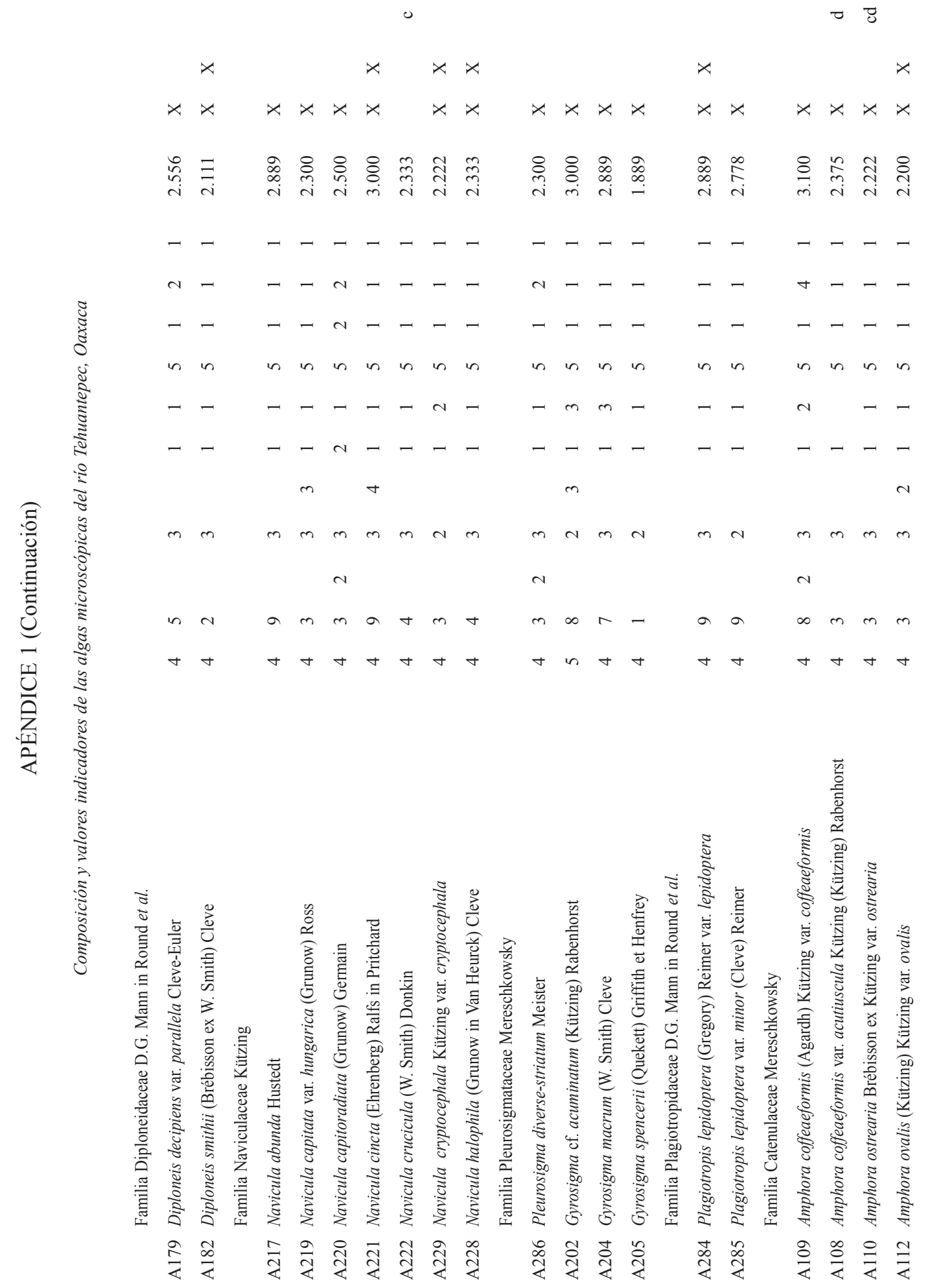




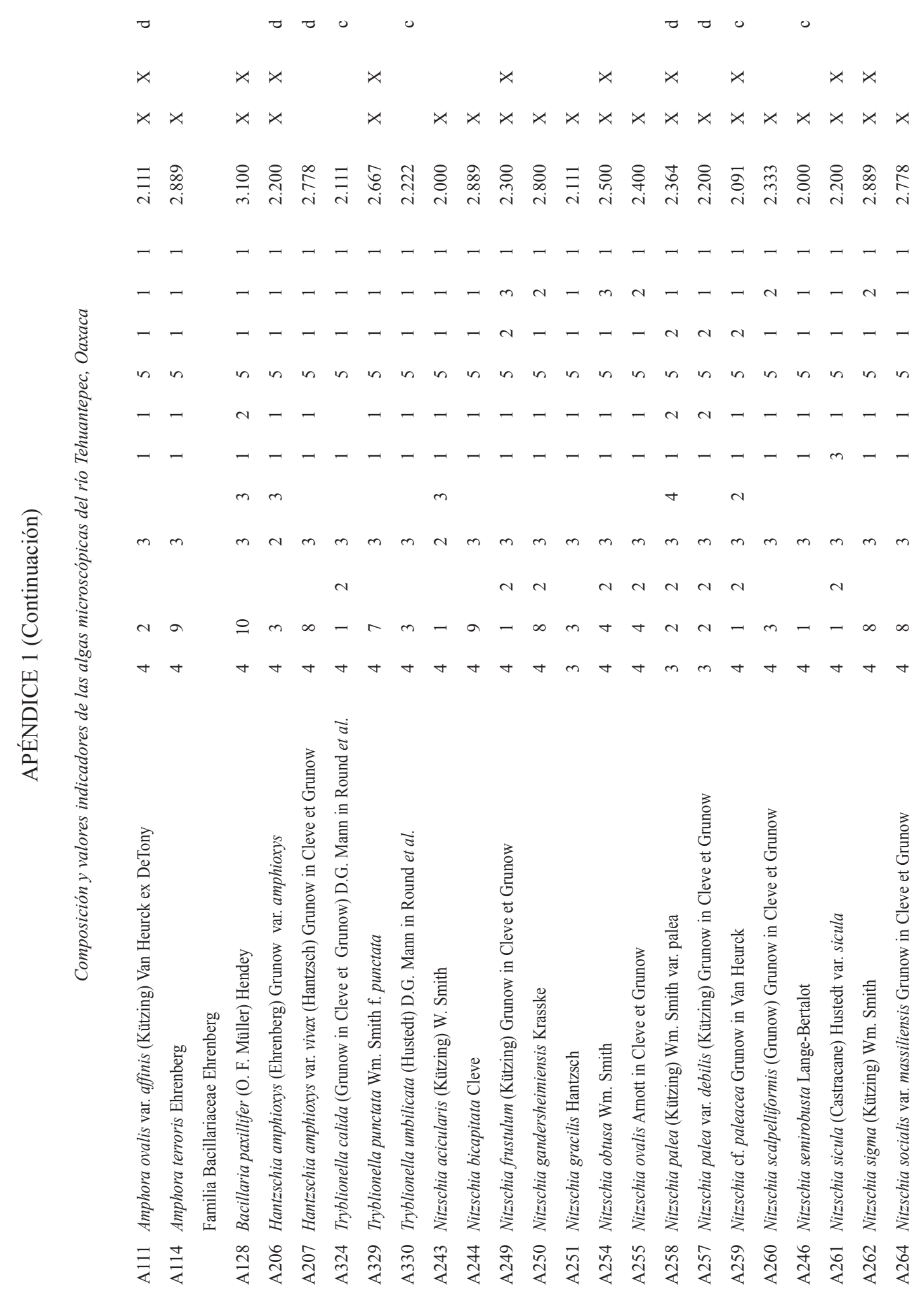




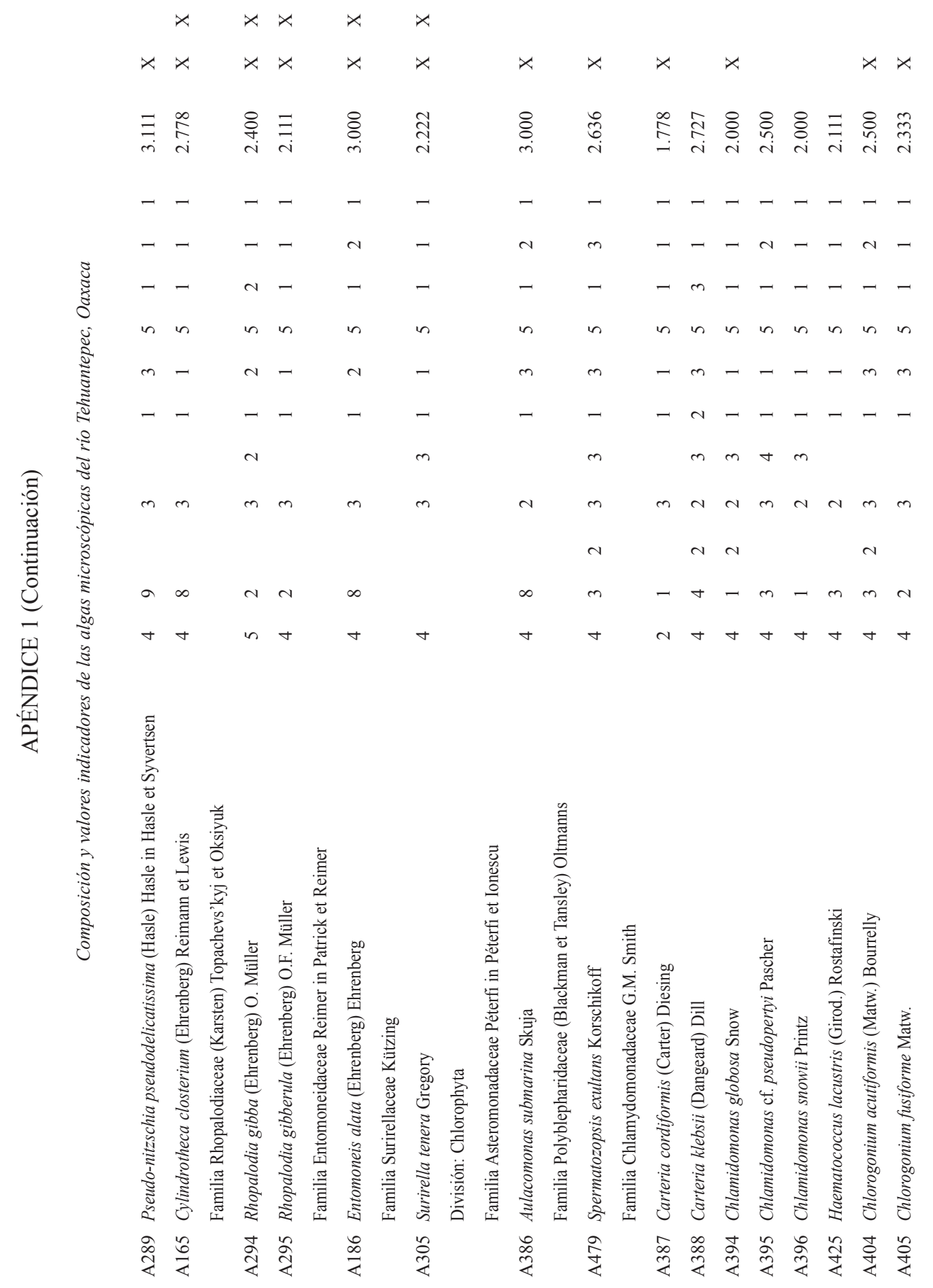




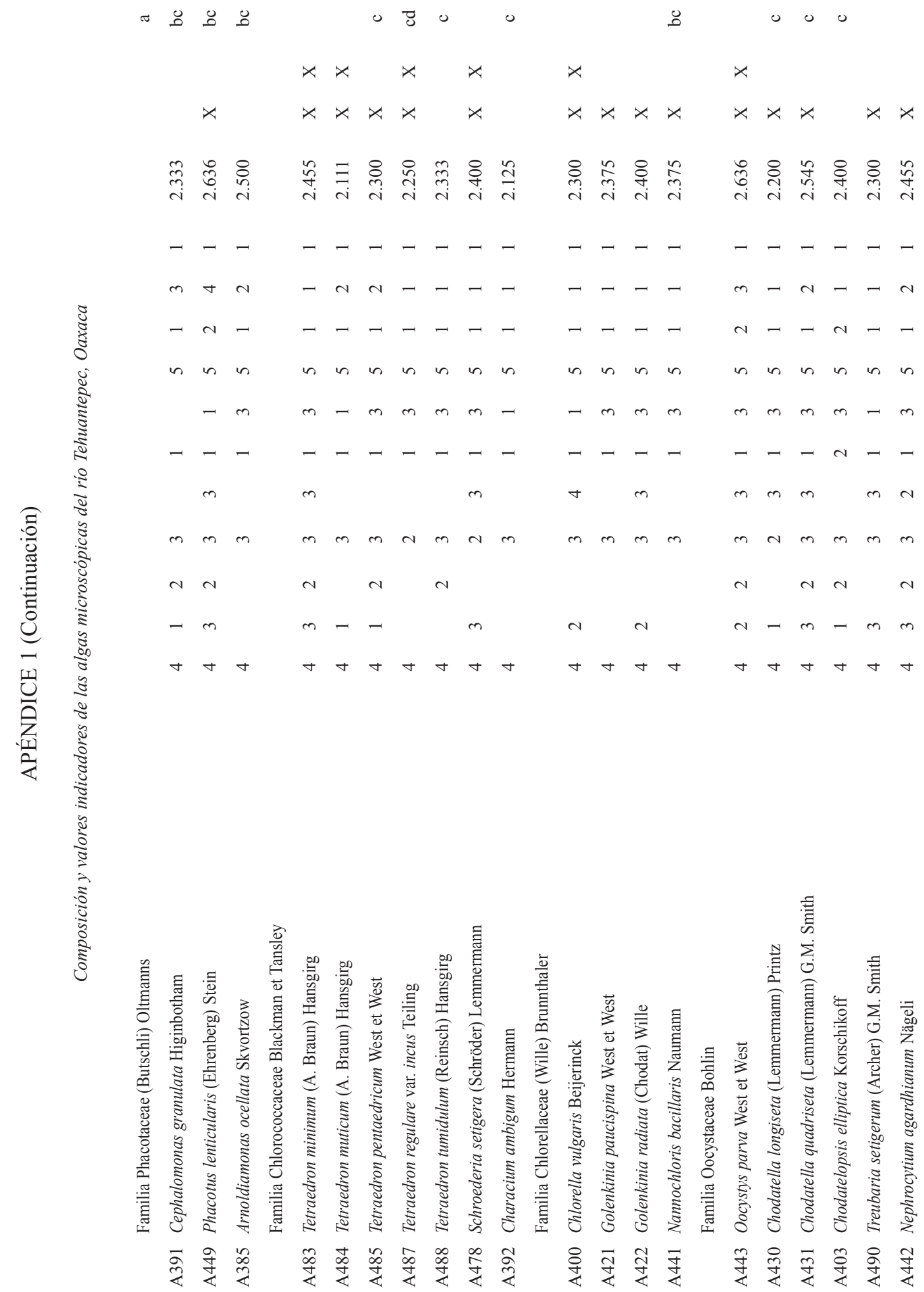




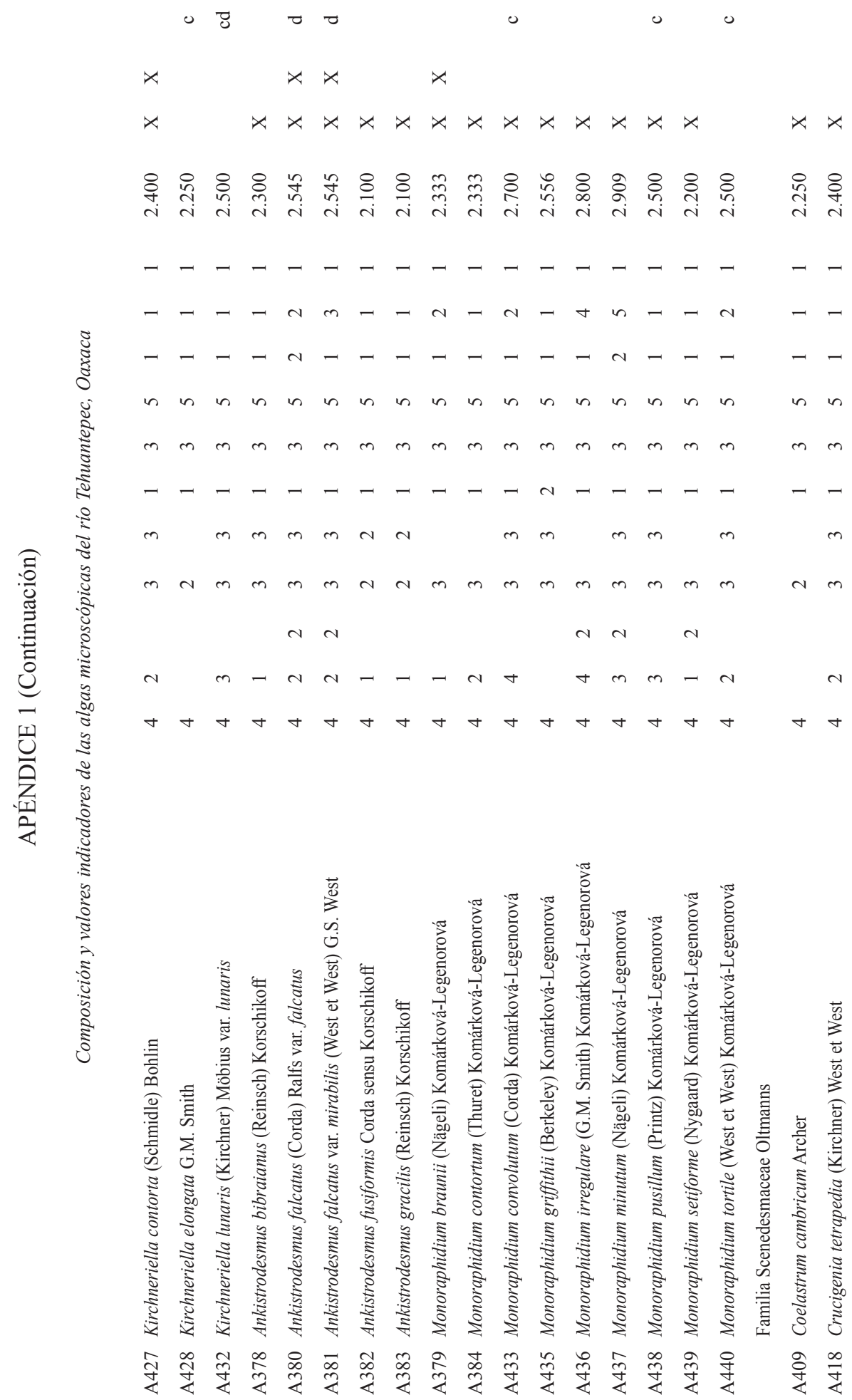




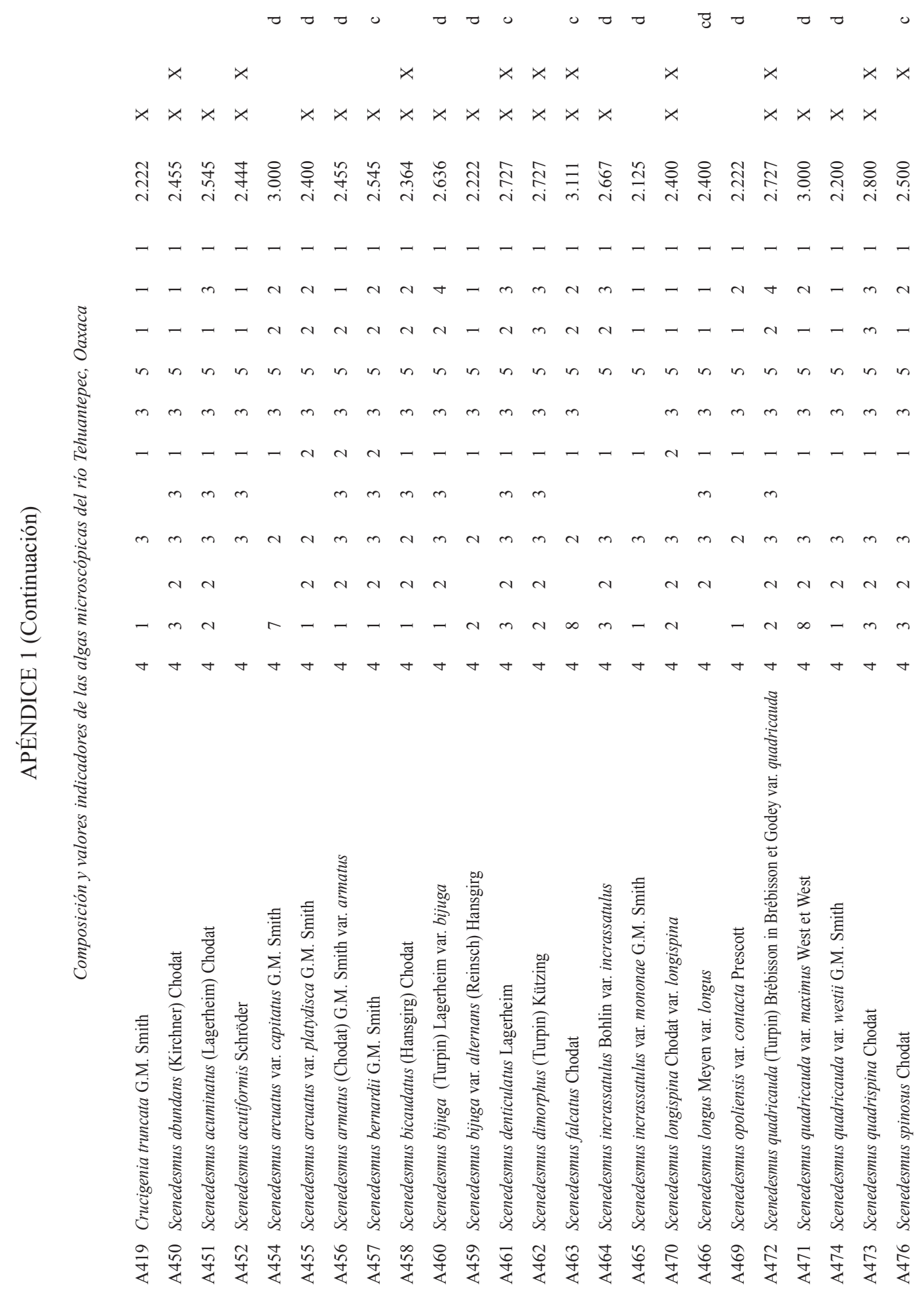




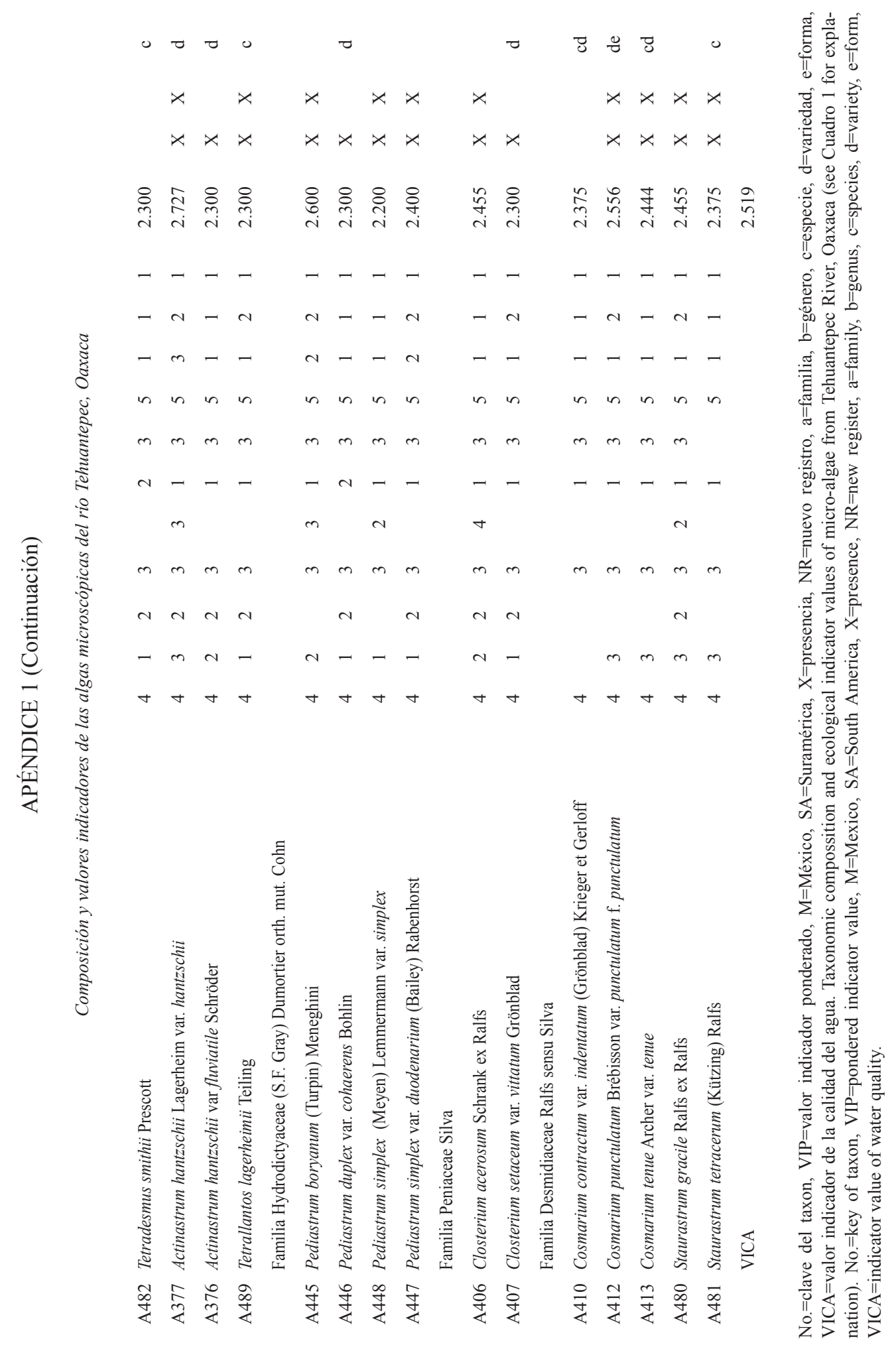

